\title{
NUMERICAL SCHEMES FOR COMPUTING DISCONTINUOUS SOLUTIONS OF THE DEGASPERIS-PROCESI EQUATION
}

\author{
G. M. COCLITE, K. H. KARLSEN, AND N. H. RISEBRO
}

\begin{abstract}
Recent work [4] has shown that the Degasperis-Procesi equation is well-posed in the class of (discontinuous) entropy solutions. In the present paper we construct numerical schemes and prove that they converge to entropy solutions. Additionally, we provide several numerical examples accentuating that discontinuous (shock) solutions form independently of the smoothness of the initial data. Our focus on discontinuous solutions contrasts notably with the existing literature on the Degasperis-Procesi equation, which seems to emphasize similarities with the Camassa-Holm equation (bi-Hamiltonian structure, integrabillity, peakon solutions, $H^{1}$ as the relevant functional space).
\end{abstract}

\section{IntRoduCtion}

In this paper we present and analyze several numerical schemes for capturing discontinuous solutions of the Degasperis-Procesi equation [8]

$$
\partial_{t} u-\partial_{t x x}^{3} u+4 u \partial_{x} u=3 \partial_{x} u \partial_{x x}^{2} u+u \partial_{x x x}^{3} u, \quad(x, t) \in \mathbb{R} \times(0, T) .
$$

We are interested in the Cauchy problem for this equation where an initial function $u_{0}$ is prescribed at time $t=0:\left.u\right|_{t=0}=u_{0}$. The Degasperis-Procesi equation is a special case of the more general equation

$$
\partial_{t} u-\partial_{t x x}^{3} u+4 \partial_{x} f(u)=\partial_{x x x}^{3} f(u),
$$

where (1.1) is recovered by choosing $f(u)=\frac{1}{2} u^{2}$. We shall concentrate on this equation, which we coin the generalized Degasperis-Procesi equation, and show that our numerical schemes converge if

$$
|f(u)| \leq \kappa u^{2} \text { for all } u \text {, for some constant } \kappa>0 .
$$

About the motivation for considering (1.2) instead of (1.1), besides the increased generality, we remark at this stage only that (1.2) will play a central role in the analysis of numerical schemes for (1.1) as well.

Degasperis and Procesi [8] studied the following six parameter family of third order dispersive equations

$$
\partial_{t} u+c_{0} \partial_{x} u+\gamma \partial_{x x x}^{3} u-\alpha^{2} \partial_{t x x}^{3} u=\partial_{x}\left(c_{1} u^{2}+c_{2}\left(\partial_{x} u\right)^{2}+c_{3} u \partial_{x x}^{2} u\right) .
$$

Using the method of asymptotic integrability, they found that three equations of this family to be asymptotically integrable up to third order: the KdV equation

Date: March 29, 2006.

2000 Mathematics Subject Classification. 35G25, 35L05, 35L65, 74S20, 65M12.

Key words and phrases. Shallow water equation, integrable equation, hyperbolic equation, discontinuous solution, weak solution, entropy condition, numerical scheme, convergence.

K. H. Karlsen is supported by an Outstanding Young Investigators Award. The authors are grateful to Hans Lundmark for interesting discussions. 
$\left(\alpha=c_{2}=c_{3}=0\right)$, the Camassa-Holm equation $\left(c_{1}=\left(2 c_{3}\right) /\left(2 \alpha^{2}\right), c_{2}=c_{3}\right)$ and the equation

$$
\partial_{t} u+\partial_{x} u+6 u \partial_{x} u+\partial_{x x x}^{3} u-\alpha^{2}\left(\partial_{t x x}^{3} u+\frac{9}{2} \partial_{x} u \partial_{x x}^{2} u+\frac{3}{2} u \partial_{x x x}^{3} u\right)=0 .
$$

By rescaling, shifting the dependent variable, and applying a Galilean boost, this transforms to $(1.1)$, see $[7,6]$ for details.

Formally, equation (1.2) is equivalent to the hyperbolic-elliptic system

$$
\partial_{t} u+\partial_{x} f(u)+\partial_{x} P=0, \quad P-\partial_{x x}^{2} P=3 f(u),
$$

which also provides the starting point for defining weak solutions of (1.2). The Helmholtz operator $\left(1-\partial_{x x}^{2}\right)^{-1}$ can be written as a convolution

$$
\left(1-\partial_{x x}^{2}\right)^{-1}(g)(x)=\frac{1}{2} \int_{\mathbb{R}} e^{-|x-y|} g(y) d y,
$$

and consequently

$$
P(x, t)=\frac{3}{2} \int_{\mathbb{R}} e^{-|x-y|} f(u(y, t)) d y .
$$

The Degasperis-Procesi equation (1.1) has a form similar to the Camassa-Holm shallow water wave equation (see, e.g., $[2,5,10,19]$ and the references therein for more information about this equation), and many authors have emphasized that (1.1) share several properties with the Camassa-Holm equation such as biHamiltonian structure, integrabillity, exact solutions that are a superposition of multipeakons, and $H^{1}$ as the relevant functional space for well-posedness. Let us also mention that a simplified model for radiating gases is given by a hyperbolicelliptic system that bears some similarities with (1.4), see, e.g., [12, 13, 18].

Degasperis, Holm, and Hone [7] proved the exact integrability of (1.1) and found exact "non-smooth" multipeakon solutions. Lundmark and Szmigielski $[15,16]$ used an inverse scattering approach to determine an explicit formula for the general $n$ peakon solution of the Degasperis-Procesi equation (1.1). Mustafa [17] showed that smooth solutions to (1.1) have the infinite speed of propagation property.

Yin studied the globall well-posedness of the Degasperis-Procesi equation (1.1) in a series of papers $[20,21,22,23]$. The (weak) solutions encompassed by Yin's well-posedness theory are all $H^{1}$ regular (typical examples are the peakons), a fact that is reminiscent of the Camassa-Holm equation (see, e.g., [5]).

We refer to [10] for an overview of the Camassa-Holm, Degasperis-Procesi, and other related equations. There the authors present many numerical examples and include some comments on the relevance of these equations as shallow water models.

In a different direction, two of the authors of the present paper promoted recently [4] the view that the Degasperis-Procesi equation could admit discontinuous (shock wave) solutions and that a well-posedness theory should rely on functional spaces containing discontinuous functions. Indeed, [4] proved the existence, uniqueness, and $L^{1}$ stability of so-called entropy solutions which belong to the space $B V$ of functions of bounded variation.

Lundmark [14] recently found some explicit shock solutions of the DegasperisProcesi equation (1.1) that are entropy solutions (in the sense of [4]). A simple example of such a discontinuous solution to (1.1) is provided by

$$
u(x, t)= \begin{cases}1+\frac{1}{1+t} e^{x-t}, & x<t, \\ 1-\frac{1}{1+t} e^{-(x-t)}, & x>t,\end{cases}
$$


which corresponds to the initial data $u_{0}(x)= \begin{cases}1+e^{x}, & x<0 \\ 1-e^{-x}, & x>0\end{cases}$

Since entropy solutions are of importance to us, let us recall their definition.

Definition 1.1 (Entropy solution). A function $u(x, t)$ is an entropy solution of the generalized Degasperis-Procesi equation (1.2) with initial data $\left.u\right|_{t=0}=u_{0} \in L^{\infty}(\mathbb{R})$ provided the following two conditions hold:

i) $u \in L^{\infty}(\mathbb{R} \times(0, T))$, and

iii) for any convex $C^{2}$ entropy $\eta: \mathbb{R} \rightarrow \mathbb{R}$ with corresponding entropy flux $q: \mathbb{R} \rightarrow \mathbb{R}$ defined by $q^{\prime}(u)=\eta^{\prime}(u) f^{\prime}(u)$ there holds

$$
\partial_{t} \eta(u)+\partial_{x} q(u)+\eta^{\prime}(u) \partial_{x} P^{u} \leq 0 \text { in } \mathcal{D}^{\prime}(\mathbb{R} \times[0, T)),
$$

that is, $\forall \phi \in C_{c}^{\infty}(\mathbb{R} \times[0, T)), \phi \geq 0$,

$$
\begin{aligned}
& \int_{0}^{T} \int_{\mathbb{R}}\left(\eta(u) \partial_{t} \phi+q(u) \partial_{x} \phi-\eta^{\prime}(u) \partial_{x} P^{u} \phi\right) d x d t+\int_{\mathbb{R}} \eta\left(u_{0}(x)\right) \phi(x, 0) d x \geq 0, \\
& \text { where } \\
& P^{u}(x, t)=\frac{3}{2} \int_{\mathbb{R}} e^{-|x-y|} f(u(y, t)) d y .
\end{aligned}
$$

From (1.3) and part $i$ ) of Definition 1.1, it is clear that $\partial_{x} P^{u} \in L^{\infty}\left(\mathbb{R} \times \mathbb{R}_{+}\right)$ and thus the entropy formulation (1.6) makes sense. Another remark is that it is sufficient to verify (1.5) for the Kružkov entropies/entropy fluxes

$$
\eta(u)=|u-c|, \quad q(u)=\operatorname{sign}(u-c)(f(u)-f(c)), \quad c \in \mathbb{R} .
$$

Using the Kružkov entropies it can be seen that the following weak formulation (1.6) is a consequence of the entropy formulation (1.5): $\forall \phi \in C_{c}^{\infty}(\mathbb{R} \times[0, T))$,

$$
\int_{0}^{T} \int_{\mathbb{R}}\left(u \partial_{t} \phi+f(u) \partial_{x} \phi-\partial_{x} P^{u} \phi\right) d x d t+\int_{\mathbb{R}} u_{0}(x) \phi(x, 0) d x=0 .
$$

Regarding the $L^{1}$ stability (and thus uniqueness) of entropy solutions to the generalized Degasperis-Procesi equation, the following result is proved in [4]:

Theorem 1.1 ( $L^{1}$ stability). Suppose $f \in C^{1}(\mathbb{R})$. Let $u, v \in C\left([0, T] ; L^{1}(\mathbb{R})\right)$ be two entropy solutions of the generalized Degasperis-Procesi equation (1.2) with initial data $u_{0}, v_{0} \in L^{1}(\mathbb{R}) \cap L^{\infty}(\mathbb{R})$, respectively. Then for any $t \in(0, T)$

$$
\|u(t, \cdot)-v(t, \cdot)\|_{L^{1}(\mathbb{R})} \leq e^{C t}\left\|u_{0}-v_{0}\right\|_{L^{1}(\mathbb{R})},
$$

where

$$
C=\frac{3}{2}\left\|f^{\prime}\right\|_{L^{\infty}([-M, M])}, \quad M=\max \left[\|u\|_{L^{\infty}((0, T) \times \mathbb{R})},\|v\|_{L^{\infty}((0, T) \times \mathbb{R})}\right] .
$$

Consequently, there exists at most one entropy solution to (1.2) with initial data $\left.u\right|_{t=0}=u_{0} \in L^{1}(\mathbb{R}) \cap L^{\infty}(\mathbb{R})$.

The existence of entropy solutions to (1.2) is proved in [4] under the assumption that $u_{0} \in L^{1}(\mathbb{R}) \cap B V(\mathbb{R})$. These entropy solutions $u$ satisfy

$$
u \in L^{\infty}\left(0, T ; L^{2}(\mathbb{R})\right), \quad u \in L^{\infty}\left(0, T ; L^{1}(\mathbb{R}) \cap B V(\mathbb{R})\right),
$$

where the latter $L^{1} \cap B V$ bound, which depends on $T$, is a consequence of the former $L^{2}$ bound. The $L^{\infty}$ bound required by Definition 1.1 is a consequence of 
the $B V$ bound. The $L^{2}$ bound, which is independent of $T$, is at the heart of the matter in [4], and formally comes from the conservation law

$$
\partial_{t}\left(\left(\partial_{x x}^{2} v\right)^{2}+5\left(\partial_{x} v\right)^{2}+4 v^{2}\right)+\partial_{x}\left(\frac{2}{3} u^{3}+4 v w+\partial_{x} v \partial_{x} w-4 u^{2} v\right)=0
$$

where $v:=\left(4-\partial_{x x}^{2}\right)^{-1}(u), w:=\left(1-\partial_{x x}^{2}\right)^{-1}\left(u^{2}\right)$. A consequence of this equation is that $v(t, \cdot) \in H^{2}(\mathbb{R})$ and thereby $u(t, \cdot) \in L^{2}(\mathbb{R})$, for any $t \geq 0$.

Let us now turn to the topic of the present paper, namely numerical schemes for capturing discontinuous entropy solutions of the generalized Degasperis-Procesi equation (1.2), which is to be interpreted in the sense of (1.4). First of all, the form of the equation (1.4) resembles a model of two phase flow in porous media. In a simplified form this model reads

$$
\left.\begin{array}{r}
\partial_{t} u+\operatorname{div}(\mathbf{v} F(u))=0 \\
\operatorname{div}(\Lambda(u) \nabla P)=0
\end{array}\right\} \quad(\mathbf{x}, t) \in U \times(0, T],
$$

where $U$ is a bounded domain in $\mathbb{R}^{2}$ or $\mathbb{R}^{3}$, and $\Lambda$ is a given function of $u$, and $\mathbf{v}=\Lambda(u) \nabla P$. Here $u \in[0,1]$ denotes the saturation of one of the phases, and the flux function $F$ is an increasing function $F:[0,1] \mapsto[0,1]$ with $F(0)=0$ and $F(1)=1$. The variable $P$ represents the pressure. Since (1.10) is assumed to hold in a bounded domain, it is necessary to prescribe boundary values, at least for the total velocity $\mathbf{v}$, as well as initial values for $u$. We remark that the well-posedness of the boundary/initial value problem for (1.10) is open, although results exist when capillary pressure forces (a degenerate diffusion operator is added to the equations), see [3]. Numerically, (1.10) is often "solved" using an "operator splitting" strategy in which the elliptic equation for $P$ (the pressure equation) is solved to give a pressure which is used for the hyperbolic equation (the saturation equation), see for example [1] and the references therein. The rationale behind this is that the time scales in the pressure and saturation equations are sufficiently different, so that (presumably) the total velocity will change much slower than the saturation.

This type of operator splitting inspired the construction of the numerical schemes presented in this paper. Informally, this splitting approximation is constructed as follows. Let $S_{t}^{g}$ denote the solution operator for the balance law

$$
\partial_{t} v+\partial_{x} f(v)+g(x)=0, \quad v(x, 0)=v_{0}(x),
$$

i.e., we formally write the entropy solution $v(x, t)$ as

$$
v(x, t)=S_{t}^{g}\left(u_{0}\right)(x) .
$$

Then we can define a sequence of functions $\left\{u^{n}\right\}_{n \geq 0}$ by $u^{0}:=u_{0}$ and

$$
u^{n}:=S_{\Delta t}^{g_{n-1}}\left(u^{n-1}\right), \quad g_{n-1}:=\partial_{x} P^{n-1}, \quad P^{n-1}-P_{x x}^{n-1}=3 f\left(u^{n-1}\right) .
$$

The resulting approximation $u_{\Delta t}=u_{\Delta t}(x, t)$ is defined as

$$
u_{\Delta t}(\cdot, t)=u^{n} \quad \text { for } n \Delta t \leq t<(n+1) \Delta t .
$$

Here $\Delta t$ is a small parameter (the "time step"). The hope is that $u_{\Delta t} \rightarrow u$ as $\Delta t \rightarrow 0$ in some reasonable topology, and that $u$ is the entropy solution to (1.4). In this paper we establish this convergence. Additionally, we establish convergence of fully discrete numerical schemes constructed using the splitting approach and monotone difference schemes to discretize (1.11) and the equation in (1.12) for $P_{n}$. 
To make the topology for the conservation law and the elliptic equation for $P$ "compatible", we assume that $f$ is globally Lipschitz continuous in the convergence analysis of the numerical schemes. It is difficult at the discrete level to establish (1.9), which would have provided us with a uniform $L^{2}$ estimate on the numerical approximations. This in turn would have implied uniform $B V, L^{1}, L^{\infty}$ estimates, and compactness of the numerical approximations would then have followed. On the other hand, if the nonlinearity $f$ is globally Lipschitz continuous, then $B V, L^{1}, L^{\infty}$ estimates can be derived directly without going via an $L^{2}$ estimate. Thanks to the uniqueness of entropy solutions (Theorem 1.1), we can without loss of generality assume that $f$ is globally Lipschitz continuous. If not, we can replace the function $f$ by a globally Lipschitz continuous function, call it $f_{L}$, such that $f_{L}$ coincides with $f$ on $[-L, L]$ for $L>0$ and $\left|f_{L}(u)\right| \leq \kappa u^{2}$ for all $u$ with $\kappa$ given in (1.3). Let $u_{L}$ be the entropy solution of (1.2) with $f$ replaced by $f_{L}$ and initial data $\left.u_{L}\right|_{t=0}=u_{0}$. It follows from the results in [4] that the $L^{1}, L^{2}, L^{\infty}, B V$ bounds on $u_{L}$ coincides with those attached to $u$. Choosing $L>M$ we conclude by the uniqueness of entropy solutions that the functions $u$ and $u_{L}$ must coincide. In other words, thanks to the well-posedness analysis in [4] and without any loss of generality, we can restrict ourselves to the case where $f$ in (1.2) is globally Lipschitz continuous.

The rest of this paper is organized as follows: In Section 2 we establish some preliminary estimates for the balance equation where $P$ is given by the initial data only. Furthermore, as a "warm-up", we establish the convergence of a monotone difference scheme for this balance law. In Section 3 we define the operator splitting method. First we establish convergence of a semi-discrete splitting approximation, and then we proceed to show the convergence of fully discrete schemes. Finally, in Section 4 we present some numerical results. These examples show that shock solutions form independently of the smoothness of the initial data, and hence that the entropy solution framework is reasonable for this type of equations.

\section{Preliminary estimates for balance laws}

As a starting point we consider the Cauchy problem for the conservation law with a source (which in this case is determined by the initial condition)

$$
\begin{cases}\partial_{t} u+\partial_{x} f(u)+\partial_{x} P=0, & x \in \mathbb{R}, 0<t<T, \\ -\partial_{x x}^{2} P+P=3 f\left(u_{0}\right), & x \in \mathbb{R}, \\ u(x, 0)=u_{0}(x), & x \in \mathbb{R},\end{cases}
$$

where we shall assume that the initial condition satisfies

$$
u_{0} \in L^{1}(\mathbb{R}) \cap B V(\mathbb{R}) .
$$

As explained in the introduction, it is sufficient to consider nonlinearities $f$ that are globally Lipschitz continuous. More precisely, we assume

$$
f \in C^{1}(\mathbb{R}), \quad f(0)=0, \quad\left|f(\xi)-f\left(\xi^{\prime}\right)\right| \leq L\left|\xi-\xi^{\prime}\right|,
$$

for all $\xi, \xi^{\prime} \in \mathbb{R}$ and some positive constant $L>0$.

2.1. Estimates on the exact solutions. First we collect some estimates satisfied by the "source" $P$ in (2.1).

Lemma 2.1 (Estimates on $P$ ). Assume that (2.2) and (2.3) are satisfied. Then

$$
\|P\|_{L^{\infty}(\mathbb{R})},\left\|\partial_{x} P\right\|_{L^{\infty}(\mathbb{R})} \leq \frac{3}{2} L\left\|u_{0}\right\|_{L^{1}(\mathbb{R})},
$$




$$
\begin{aligned}
\|P\|_{L^{1}(\mathbb{R})},\left\|\partial_{x} P\right\|_{L^{1}(\mathbb{R})} & \leq 3 L\left\|u_{0}\right\|_{L^{1}(\mathbb{R})} \\
\left\|\partial_{x x}^{2} P\right\|_{L^{1}(\mathbb{R})} & \leq 6 L\left\|u_{0}\right\|_{L^{1}(\mathbb{R})} \\
\left\|\partial_{x x}^{2} P\right\|_{L^{\infty}(\mathbb{R})} & \leq \frac{3}{2} L\left\|u_{0}\right\|_{L^{1}(\mathbb{R})}+3 L\left|u_{0}\right|_{B V(\mathbb{R})} .
\end{aligned}
$$

Proof. The proof follows from the observation that $e^{-|x|} / 2$ is the Green's function of the differential operator $1-\partial_{x x}^{2}$, and thus we have that

$$
\begin{aligned}
P(x) & =\frac{3}{2} \int_{\mathbb{R}} e^{-|x-y|} f\left(u_{0}(y)\right) d y, \quad x \in \mathbb{R}, \\
\partial_{x} P(x) & =\frac{3}{2} \int_{\mathbb{R}} e^{-|x-y|} \operatorname{sign}(y-x) f\left(u_{0}(y)\right) d y, \quad x \in \mathbb{R} .
\end{aligned}
$$

Since $e^{-|x|} \leq 1, \int_{\mathbb{R}} e^{-|x-y|} d y=2$, for all $x \in \mathbb{R}$, by $(2.3)$,

$$
\begin{aligned}
|P(x)|,\left|\partial_{x} P(x)\right| & \leq \frac{3}{2} \int_{\mathbb{R}}\left|f\left(u_{0}(y)\right)\right| d y \leq \frac{3}{2} L \int_{\mathbb{R}}\left|u_{0}(y)\right| d y, \quad x \in \mathbb{R}, \\
\int_{\mathbb{R}}|P(x)| d x, \int_{\mathbb{R}}\left|\partial_{x} P(x)\right| d x & \leq 3 \int_{\mathbb{R}}\left|f\left(u_{0}(y)\right)\right| d y \leq 3 L \int_{\mathbb{R}}\left|u_{0}(y)\right| d y,
\end{aligned}
$$

which give (2.4), (2.5), respectively. Using $\partial_{x x}^{2} P=P-3 f\left(u_{0}\right)$ gives the final estimates.

Lemma 2.1 gives us the necessary control to prove some estimates for the entropy solution $u$ of the conservation law (2.1) with source $\partial_{x} P$. We collect these in the following lemma, whose proof is standard and therefore omitted (see [9]).

Lemma 2.2 (Estimates on $u$ ). Assume that (2.2) and (2.3) hold. Then for each $t \in(0, T)$

$$
\begin{gathered}
\|u(\cdot, t)\|_{L^{1}(\mathbb{R})} \leq(1+3 L t)\left\|u_{0}\right\|_{L^{1}(\mathbb{R})}, \\
\|u(\cdot, t)\|_{L^{\infty}(\mathbb{R})} \leq|u(\cdot, t)|_{B V(\mathbb{R})} \leq\left|u_{0}\right|_{B V(\mathbb{R})}+6 L t\left\|u_{0}\right\|_{L^{1}(\mathbb{R})} .
\end{gathered}
$$

Additionally, there is a constant $C_{T}$, independent of the discretization parameters, such that

$$
\|u(\cdot, t)-u(\cdot, s)\|_{L^{1}(\mathbb{R})} \leq C_{T}|t-s|, \quad \forall t, s \in[0, T] .
$$

2.2. Finite difference schemes. We shall analyze the following finite difference discretization of (2.1):

$$
\begin{aligned}
& \frac{U_{j}^{n+1}-U_{j}^{n}}{\Delta \tau}+D_{-} F\left(U^{n} ; j\right)+D P_{j}=0, \quad j \in \mathbb{Z}, n \in\{0, \ldots, N-1\}, \\
& U_{j}^{0}=\frac{1}{\Delta x} \int_{\left(j+\frac{1}{2}\right) \Delta x}^{\left(j-\frac{1}{2}\right) \Delta x} u_{0}(x) d x, \quad j \in \mathbb{Z}, \\
& -D_{+} D_{-} P_{j}+P_{j}=3 f\left(U_{j}^{0}\right), \quad j \in \mathbb{Z},
\end{aligned}
$$

where $\Delta \tau, \Delta x>0$ denote the temporal and spatial discretization parameters, respectively, while $N$ is the smallest integer such that $N \Delta t \geq T$. Moreover, 
$D_{+}, D_{-}, D$ denote the forward, backward, and central spatial difference operators, respectively:

$$
\begin{aligned}
D_{+} \xi_{j} & :=\frac{\xi_{j+1}-\xi_{j}}{\Delta x}, \quad D_{-} \xi_{j}:=\frac{\xi_{j}-\xi_{j-1}}{\Delta x}, \\
D \xi_{j} & :=\frac{D_{+} \xi_{j}+D_{-} \xi_{j}}{2}=\frac{\xi_{j+1}-\xi_{j-1}}{2 \Delta x} .
\end{aligned}
$$

The ratio $\Delta \tau / \Delta x=\lambda$ is assumed to be constant. We use the following notation for the numerical flux function $F$ :

$$
F(U ; j)=F\left(U_{j-p}, U_{j-p+1}, \ldots, U_{j+q}\right),
$$

for some integers $p, q \geq 0$. The numerical flux is assumed to be consistent, i.e.,

$$
F(u, \ldots, u)=f(u),
$$

and such that for the conservation law without source the scheme would be TVD. This means that

$$
\sum_{j=-\infty}^{\infty}\left|\xi_{j}-\xi_{j-1}-\lambda(F(\xi ; j)+F(\xi ; j-1))\right| \leq\left|\left\{\xi_{j}\right\}_{j}\right|_{b v},
$$

for sufficiently small $\lambda$, and where $\left\{\xi_{j}\right\}_{j \in \mathbb{Z}}$ is any real sequence. Throughout this paper we use the notations

$$
\left|\left\{\xi_{j}\right\}_{j}\right|_{b v}=\sum_{j}\left|\xi_{j}-\xi_{j-1}\right|, \quad\left\|\left\{\xi_{j}\right\}_{j}\right\|_{\ell^{1}}=\Delta x \sum_{j}\left|\xi_{j}\right|, \quad\left\|\left\{\xi_{j}\right\}_{j}\right\|_{\ell^{\infty}}=\sup _{j}\left|\xi_{j}\right| .
$$

Recall that for (2.12) to hold, we must assume that the CFL-condition

$$
\Delta t \leq \lambda \Delta x
$$

holds, for a specific constant $\lambda$ determined by $f$ and $u_{0}$ [9]. In this paper, we assume that the TVD property (2.12) is valid.

There are numerous examples of numerical fluxes, see for example [9]. For the numerical examples in this paper we use the Engquist-Osher flux

$$
F(u, v)=\frac{1}{2}\left(f(u)+f(v)-\int_{u}^{v}\left|f^{\prime}(w)\right| d w\right) .
$$

Lemma 2.3 (Estimates on $P_{j}$ ). Assume that (2.2) and (2.3) hold. Then there exists a positive constant $C_{1}$, independent of $f, u_{0}, \Delta \tau, \Delta x$, such that

$$
\begin{array}{r}
\left\|\left\{P_{j}\right\}_{j}\right\|_{\ell^{\infty}},\left\|\left\{D_{-} P_{j}\right\}_{j}\right\|_{\ell^{\infty}},\left\|\left\{D_{+} P_{j}\right\}_{j}\right\|_{\ell^{\infty}},\left\|\left\{D P_{j}\right\}_{j}\right\|_{\ell^{\infty}} \leq C_{1} L\left\|u_{0}\right\|_{L^{1}(\mathbb{R})}, \\
\left\|\left\{P_{j}\right\}_{j}\right\|_{\ell^{1}},\left\|\left\{D_{-} P_{j}\right\}_{j}\right\|_{\ell^{1}},\left\|\left\{D_{+} P_{j}\right\}_{j}\right\|_{\ell^{1}},\left\|\left\{D P_{j}\right\}_{j}\right\|_{\ell^{1}} \leq C_{1} L\left\|u_{0}\right\|_{L^{1}(\mathbb{R})}, \\
\left\|\left\{D_{+} D_{-} P_{j}\right\}_{j}\right\|_{\ell^{1}} \leq C_{1} L\left\|u_{0}\right\|_{L^{1}(\mathbb{R})} .
\end{array}
$$

Proof. The discrete equation for $P_{j}$ can be solved explicitly:

$$
P_{j}=3 h \sum_{i=-\infty}^{\infty} e^{-\kappa|j-i|} f\left(U_{i}^{0}\right), \quad j \in \mathbb{Z}
$$

where

$$
h:=\left(1+2 \frac{1-e^{-\kappa}}{(\Delta x)^{2}}\right)^{-1}, \quad \kappa:=\ln \left(1+\frac{(\Delta x)^{2}}{2}+\frac{\Delta x}{2} \sqrt{4+(\Delta x)^{2}}\right)
$$


These formulas can be derived by applying the discrete Fourier transform to the equation determining $P_{j}$. Observe that

$$
h=\mathcal{O}(\Delta x), \quad \frac{e^{\kappa}-1}{\Delta x}, 1-e^{-\kappa} \leq 1+\mathcal{O}(\Delta x), \quad\left|e^{-\kappa}-1\right| \leq \Delta x .
$$

Then we can proceed as in the continuous case:

$$
\begin{aligned}
D_{-} P_{j} & =\frac{P_{j}-P_{j-1}}{\Delta x}=3 h \sum_{i=-\infty}^{\infty} \frac{e^{-\kappa|i-j|}-e^{-\kappa|i-j+1|}}{\Delta x} f\left(U_{i}^{0}\right) \\
& =3 h \sum_{i=j}^{\infty} \frac{e^{-\kappa(i-j)}-e^{-\kappa(i-j+1)}}{\Delta x} f\left(U_{i}^{0}\right)+3 h \sum_{i=-\infty}^{j-1} \frac{e^{\kappa(i-j)}-e^{\kappa(i-j+1)}}{\Delta x} f\left(U_{i}^{0}\right) \\
& =3 h \sum_{i=j}^{\infty} e^{-\kappa(i-j)} \frac{1-e^{-\kappa}}{\Delta x} f\left(U_{i}^{0}\right)+3 h \sum_{i=-\infty}^{j-1} e^{\kappa(i-j)} \frac{1-e^{\kappa}}{\Delta x} f\left(U_{i}^{0}\right) .
\end{aligned}
$$

Using (2.3) and (2.19), for each $j \in \mathbb{Z}$ and some constants $c_{1}, c_{2}, c_{3}>0$,

$$
\begin{aligned}
\left|P_{j}\right| & \leq 3 h \sum_{i=-\infty}^{\infty} e^{-\kappa|j-i|}\left|f\left(U_{i}^{0}\right)\right| \leq 3 h \sum_{i=-\infty}^{\infty}\left|f\left(U_{i}^{0}\right)\right| \leq 3 h L \sum_{i=-\infty}^{\infty}\left|U_{i}^{0}\right| \\
& \leq c_{1} L \Delta x \sum_{i=-\infty}^{\infty}\left|U_{i}^{0}\right| \leq c_{1} L\left\|u_{0}\right\|_{L^{1}(\mathbb{R})}, \\
\left|D_{-} P_{j}\right| & \leq 3 h \sum_{i=j}^{\infty} e^{-\kappa(i-j)} \frac{\left|1-e^{-\kappa}\right|}{\Delta x}\left|f\left(U_{i}^{0}\right)\right|+3 h \sum_{i=-\infty}^{j-1} e^{\kappa(i-j)} \frac{\left|1-e^{\kappa}\right|}{\Delta x}\left|f\left(U_{i}^{0}\right)\right| \\
& \leq 3 h c_{2} \sum_{i=-\infty}^{\infty} e^{-\kappa|i-j|}\left|f\left(U_{i}^{0}\right)\right| \leq 3 h c_{2} L \sum_{i=-\infty}^{\infty}\left|U_{i}^{0}\right| \\
& \leq 3 c_{3} L \Delta x \sum_{i=-\infty}^{\infty}\left|U_{i}^{0}\right| \leq 3 c_{3} L\left\|u_{0}\right\|_{L^{1}(\mathbb{R})} .
\end{aligned}
$$

The remaining estimates are proved in the same way.

Now we have enough estimates on the discrete source term $D P_{j}$ to establish estimates on $U_{j}^{n}$ implying compactness.

Lemma 2.4 (Estimate on $\left.U_{j}^{n}\right)$. Assume that (2.2), (2.3), and (2.12) hold. Then

$$
\begin{gathered}
\left\|\left\{U_{j}^{n}\right\}_{j}\right\|_{\ell^{1}} \leq\left(1+C_{1} \operatorname{Ln} \Delta \tau\right)\left\|u_{0}\right\|_{L^{1}(\mathbb{R})}, \\
\left\|\left\{U_{j}^{n}\right\}_{j}\right\|_{\ell^{\infty}} \leq\left|\left\{U_{j}^{n}\right\}_{j}\right|_{b v} \leq\left|\left\{U_{j}^{0}\right\}_{j}\right|_{b v}+C_{1} L n \Delta \tau\left\|u_{0}\right\|_{L^{1}(\mathbb{R})},
\end{gathered}
$$

for each $n \in\{0, \ldots, N\}$, where $C_{1}$ is given in Lemma 2.3. Additionally, there exists a constant $C_{T, L}$, independent of the discretization parameters, such that

$$
\left\|\left\{U_{j}^{n}\right\}_{j}-\left\{U_{j}^{m}\right\}_{j}\right\|_{\ell^{1}} \leq C_{T, L} \Delta t|n-m|, \quad n, m \in\{0, \ldots, N\} .
$$

Proof. This lemma is proved in the same ways as the corresponding result for a conservation law with a source [9]. 
Define the piecewise constant function $u_{\Delta \tau}$ by

$$
u_{\Delta \tau}(x, t):=\sum_{n \geq 0, j \in \mathbb{Z}} I_{j}^{n}(x, t) U_{j}^{n},
$$

where $I_{j}^{n}(x)=I_{j}(x) I^{n}(t)$, and $I^{n}$ is the characteristic function of the interval $[n \Delta \tau,(n+1) \Delta \tau)$ and $I_{j}$ of the interval $[(j-1 / 2) \Delta x,(j+1 / 2) \Delta x)$. Similarly we define the piecewise linear function $P_{\Delta x}$ by

$$
P_{\Delta \tau}(x)=\int^{x} \sum_{j \in \mathbb{Z}} D P_{j} I_{j}(y) d y .
$$

Theorem 2.1 (Convergence). Assume that (2.2), (2.3), and (2.12) hold. Then as $\Delta \tau \rightarrow 0$

$$
u_{\Delta \tau} \rightarrow u \text { in } L^{p}(\mathbb{R} \times[0, T]) \forall p<\infty \text { and a.e. in } \mathbb{R} \times[0, T],
$$

where $u$ is the unique entropy solution to (2.1) in the sense of Kružkov (see [9, Section 2.1]). We also have that

$$
D P_{\Delta \tau} \rightarrow \partial_{x} P \text { a.e. in } \mathbb{R} \times \mathbb{R}_{+} \text {as } \Delta \tau \rightarrow 0,
$$

where $P-\partial_{x x}^{2} P=3 f\left(u_{0}\right)$

Proof. Due to (2.21) and (2.22),

$$
\begin{aligned}
& \left\|u_{\Delta \tau}(\cdot, t)\right\|_{L^{1}(\mathbb{R})} \leq\left(1+C_{1} L t\right)\left\|u_{0}\right\|_{L^{1}(\mathbb{R})}, \\
& \left\|u_{\Delta \tau}(\cdot, t)\right\|_{L^{\infty}(\mathbb{R})} \leq\left|u_{\Delta \tau}(\cdot, t)\right|_{B V(\mathbb{R})} \leq\left|\left\{U_{j}^{0}\right\}_{j}\right|_{b v}+L t C_{1}\left\|u_{0}\right\|_{L^{1}(\mathbb{R})},
\end{aligned}
$$

for any $t \in(0, T)$. Additionally, due to $(2.23)$, there is a constant $C_{T, L}$, independent of the discretization parameters, such that

$$
\left\|u_{\Delta \tau}(\cdot, t)-u_{\Delta \tau}(\cdot, t)\right\|_{L^{1}(\mathbb{R})} \leq C_{T, L}|t-s|, \quad t, s \in[0, T] .
$$

Using a standard argument (see, e.g., [9]), there exists a limit function $u$ and a sequence $\left\{\Delta \tau_{l}\right\}_{l}$, with $\Delta \tau_{l} \rightarrow 0$ as $l \rightarrow \infty$, such that

$$
\begin{aligned}
& u_{\Delta \tau_{l}} \rightarrow u \text { in } L^{p}(\mathbb{R} \times[0, T]) \forall p<\infty \text { and a.e. in } \mathbb{R} \times[0, T], \\
& \|u(\cdot, t)\|_{L^{1}(\mathbb{R})},\|u(\cdot, t)\|_{L^{\infty}(\mathbb{R})},|u(\cdot, t)|_{B V(\mathbb{R})} \leq C_{T, L}, \quad \text { for each } t \in(0, T),
\end{aligned}
$$

where $C_{T, L}$ is a finite constant. In addition, $u \in C\left([0, T] ; L^{1}(\mathbb{R})\right)$.

Next we prove (2.27) which will ensure that we have the right source term in the limit in the entropy formulation of the conservation law. Observe that (see (2.17))

$$
P_{\Delta \tau_{l}}=G_{\Delta \tau_{l}} \star\left(3 f\left(u_{\Delta \tau_{l}}\right)\right), \quad P=G \star(3 f(u)),
$$

where

$$
G_{\Delta \tau_{l}}(x)=\frac{h}{\Delta x_{l}} e^{-\kappa\left|\left[\frac{x}{\Delta x_{l}}\right]\right|}, \quad G(x)=\frac{e^{-|x|}}{2}, \quad x \in \mathbb{R},
$$

where $\star$ denotes convolution in the $x$ variable and $[z]$ denotes the the integer part of $z$. By (2.28), (2.29), and the dominated convergence theorem, we have only to prove that

$$
G_{\Delta \tau_{l}}(x) \rightarrow G(x), \quad \text { for each } x \in \mathbb{R} \text { as } l \rightarrow \infty,
$$

Due to (2.18) and (2.19) we have

$$
\lim _{l \rightarrow \infty} \frac{h}{\Delta x_{l}}=\lim _{l \rightarrow \infty} \frac{1}{\Delta x_{l}+2 \frac{1-e^{-\kappa}}{\Delta x_{l}}}=\frac{1}{2} .
$$


Fixing $x \in \mathbb{R}$, due to (2.18),

$$
\begin{aligned}
\lim _{l \rightarrow \infty} e^{-\kappa\left[\left|\frac{x}{\Delta x_{l}}\right|\right]} & =\lim _{l \rightarrow \infty}\left(e^{\kappa}\right)^{-\left|\left[\frac{x}{\Delta x_{l}}\right]\right|} \\
& =\lim _{l \rightarrow \infty}\left(1+\frac{\left(\Delta x_{l}\right)^{2}}{2}+\frac{\Delta x_{l}}{2} \sqrt{4+\left(\Delta x_{l}\right)^{2}}\right)^{-\left|\left[\frac{x}{\Delta x_{l}}\right]\right|} \\
& =\lim _{l \rightarrow \infty}\left(1+\Delta x_{l}\right)^{-\left|\left[\frac{x}{\Delta x_{l}}\right]\right|} \\
& =\lim _{l \rightarrow \infty}\left(1+\Delta x_{l}\right)^{-\left|\frac{x}{\Delta x_{l}}\right|}=e^{-|x|} .
\end{aligned}
$$

Since (2.31) and (2.32) give (2.30), (2.27) is proved.

Finally, we prove that $u$ is an entropy solution to (2.1). To this end, we use a standard argument. Let $k \in \mathbb{N}$. Define the discrete entropy flux

$$
\begin{aligned}
Q_{k}(\xi ; j) & :=Q_{k}\left(\xi_{j-p}, \ldots, \xi_{j+q}\right) \\
& =F(\xi \vee k ; j)-F(\xi \wedge k ; j) \\
& =F\left(\xi_{j-p} \vee k, \ldots, \xi_{j+q} \vee k\right)-F\left(\xi_{j-p} \wedge k, \ldots, \xi_{j+q} \wedge k\right), \quad\left\{\xi_{j}\right\}_{j} \subset \mathbb{R},
\end{aligned}
$$

where

$$
a \vee b:=\max \{a, b\}, \quad a \wedge b:=\min \{a, b\}, \quad a, b \in \mathbb{R} .
$$

We begin by verifying that

$$
\frac{1}{\Delta \tau}\left(\left|U_{j}^{n+1}-k\right|-\left|U_{j}^{n}-k\right|\right)+D_{-} Q_{k}\left(U^{n} ; j\right)+D P_{j} \operatorname{sign}\left(U_{j}^{n+1}-k\right) \leq 0 .
$$

To show this we distinguish two cases. If $U_{j}^{n+1} \geq k$, from (2.10) and the monotonicity of the scheme (see [9, Theorem 3.6]) we get

$$
\begin{aligned}
\left|U_{j}^{n+1}-k\right| & =U_{j}^{n+1}-k \\
& =U_{j}^{n}-k-\lambda\left(F\left(U^{n} ; j\right)-F\left(U^{n} ; j-1\right)\right)-D P_{j} \\
& \leq\left|U_{j}^{n}-k\right|-\lambda\left(Q_{k}\left(U^{n} ; j\right)-Q_{k}\left(U^{n} ; j-1\right)\right)-D P_{j} \\
& =\left|U_{j}^{n}-k\right|-\lambda\left(Q_{k}\left(U^{n} ; j\right)-Q_{k}\left(U^{n} ; j-1\right)\right)-D P_{j} \operatorname{sign}\left(U_{j}^{n+1}-k\right) .
\end{aligned}
$$

If $U_{j}^{n+1}<k$, from (2.10) and the monotonicity of the scheme (see [9, Theorem 3.6]) we get

$$
\left|U_{j}^{n+1}-k\right| \leq\left|U_{j}^{n}-k\right|-\lambda\left(Q_{k}\left(U^{n} ; j\right)-Q_{k}\left(U^{n} ; j-1\right)\right)-D P_{j} \operatorname{sign}\left(U_{j}^{n+1}-k\right),
$$

which proves $(2.34)$.

Fix $\phi \in C_{c}^{\infty}(\mathbb{R} \times[0, \infty)), \phi \geq 0$, and multiply (2.34) by $\phi(j \Delta x, n \Delta \tau)$ to get

$$
\begin{aligned}
\sum_{n=0}^{\infty} & \sum_{j=-\infty}^{\infty} \phi(j \Delta x, n \Delta \tau) \frac{\left|U_{j}^{n+1}-k\right|-\left|U_{j}^{n}-k\right|}{\Delta \tau} \\
& +\sum_{n=0}^{\infty} \sum_{j=-\infty}^{\infty} \phi(j \Delta x, n \Delta \tau) D_{-} Q_{k}\left(U^{n} ; j\right) \\
& +\sum_{n=0}^{\infty} \sum_{j=-\infty}^{\infty} \phi(j \Delta x, n \Delta \tau) D P_{j} \operatorname{sign}\left(U_{j}^{n+1}-k\right) \leq 0 .
\end{aligned}
$$


Summing by parts yields

$$
\begin{aligned}
& \sum_{n=0}^{\infty} \sum_{j=-\infty}^{\infty} \phi(j \Delta x, n \Delta \tau) \frac{\left|U_{j}^{n+1}-k\right|-\left|U_{j}^{n}-k\right|}{\Delta \tau} \\
& =-\sum_{n=1}^{\infty} \sum_{j=-\infty}^{\infty} \frac{\phi(j \Delta x, n \Delta \tau)-\phi(j \Delta x,(n-1) \Delta \tau)}{\Delta \tau}\left|U_{j}^{n}-k\right| \\
& \quad-\sum_{j=-\infty}^{\infty} \phi(j \Delta x, 0) \frac{\left|U_{j}^{0}-k\right|}{\Delta \tau}, \\
& \sum_{n=0}^{\infty} \sum_{j=-\infty}^{\infty} \phi(j \Delta x, n \Delta \tau) D_{-} Q_{k}\left(U^{n} ; j\right)=-\sum_{n=0}^{\infty} \sum_{j=-\infty}^{\infty} Q_{k}\left(U^{n} ; j\right) D_{+} \phi(j \Delta x, n \Delta \tau),
\end{aligned}
$$

hence (2.35) becomes

$$
\begin{aligned}
& -\Delta x \Delta \tau \sum_{n=1}^{\infty} \sum_{j=-\infty}^{\infty} \frac{\phi(j \Delta x, n \Delta \tau)-\phi(j \Delta x,(n-1) \Delta \tau)}{\Delta \tau}\left|U_{j}^{n}-k\right| \\
& -\Delta x \Delta \tau \sum_{n=0}^{\infty} \sum_{j=-\infty}^{\infty} Q_{k}\left(U^{n} ; j\right) D_{+} \phi(j \Delta x, n \Delta \tau) \\
& \quad+\Delta x \Delta \tau \sum_{n=0}^{\infty} \sum_{j=-\infty}^{\infty} \phi(j \Delta x, n \Delta \tau) D P_{j} \operatorname{sign}\left(U_{j}^{n+1}-k\right) \\
& -\Delta x \sum_{j=-\infty}^{\infty} \phi(j \Delta x, 0)\left|U_{j}^{0}-k\right| \leq 0 .
\end{aligned}
$$

We claim that

$$
\Delta x \sum_{j=-\infty}^{\infty}\left|Q_{k}\left(U^{n} ; j\right)-q_{k}\left(U_{j}^{n}\right)\right| \rightarrow 0, \quad \text { as } \Delta \tau \rightarrow 0 \text { for each } n \in \mathbb{N},
$$

where

$$
q_{k}(\xi):=\operatorname{sign}(\xi-k)(f(\xi)-f(k)), \quad \xi \in \mathbb{R},
$$

is the flux associated to the entropy $\xi \mapsto|\xi-k|$. By the definition of the discrete entropy flux $Q_{k}$, we get

$$
q_{k}\left(U_{j}^{n}\right)=Q_{k}\left(U_{j}^{n}, \ldots, U_{j}^{n}\right),
$$

and hence, from (2.22), there is a constant $C_{L, T}$, independent of the discretization parameters, such that

$$
\Delta x \sum_{j=-\infty}^{\infty}\left|Q_{k}\left(U^{n} ; j\right)-q_{k}\left(U_{j}^{n}\right)\right| \leq C_{T, L} \Delta x .
$$

Let us recall the following well-known fact (see for example [11]). Let $\Omega \subset \mathbb{R}^{d}$ be a bounded open set, $g \in L^{1}(\Omega)$, and suppose that $g_{\nu}(x) \rightarrow g(x)$ a.e. in $\Omega$ as $\nu \rightarrow \infty$. Then there exists an at most countable set $\Theta$ such that for any $k \in \mathbb{R} \backslash \Theta$

$$
\operatorname{sign}\left(g_{\nu}(x)-k\right) \rightarrow \operatorname{sign}(g(x)-k) \quad \text { a.e. in } \Omega .
$$


Using this fact, (2.29), (2.37), (2.27) and the dominated convergence theorem, and sending $l \rightarrow \infty$ in (2.36), we get

$$
\begin{array}{r}
-\int_{0}^{\infty} \int_{\mathbb{R}}\left(|u-k| \partial_{t} \phi+q_{k}(u) \partial_{x} \phi-\operatorname{sign}(u-k) \partial_{x} P \phi\right) d x d t \\
-\int_{\mathbb{R}}\left|u_{0}(x)-k\right| \phi(0, x) d x \leq 0 .
\end{array}
$$

for any $k \in \mathbb{R} \backslash \Theta$, where $\Theta$ is an at most countable set $\Theta$. To conclude from this that (2.39) holds for all $k \in \mathbb{R}$ we repeat the argument in [11].

Consequently, $u$ is the Kružkov entropy solution to (2.1). Finally, convergence of the whole sequence $\left\{u_{\Delta \tau}\right\}_{\Delta \tau>0}$ follows from the uniqueness of the entropy solution to $(2.1)[9$, Section 2.4].

\section{NUMERICAL SCHEMES BASED ON OPERATOR SPLITTING}

In this section we construct numerical schemes for the generalized DegaperisProcesi equation (1.2). As explained in the introduction, there is no loss of generality in assuming that nonlinearity $f$ is globally Lipschitz continuous.

The idea is to fix $P$ for a small interval $\Delta t$, and let $u$ solve the conservation law with source $(2.1)$ for $t \in[0, \Delta t)$. Then we update $P$ and proceed to the next interval. We start by doing this for the "semi-discrete" scheme, where we use exact solutions of the conservation law and of the elliptic equation.

3.1. The semi-discrete approximation. We consider the problem

$$
\begin{cases}\partial_{t} u+\partial_{x} f(u)+\partial_{x} P=0, & x \in \mathbb{R}, 0<t<T, \\ P-\partial_{x x}^{2} P=3 f(u), & x \in \mathbb{R}, 0<t<T, \\ u(x, 0)=u_{0}(x), & x \in \mathbb{R},\end{cases}
$$

where we assume that (2.2) and (2.3) hold.

We fix a positive number $\Delta t$ and set $N$ to be the smallest integer such that $N \Delta t \geq T$. Then we define two sequences $\left\{u^{n}\right\}_{n=1}^{N}$ and $\left\{v^{n}\right\}_{n=1}^{N}$ by

$$
\begin{cases}\partial_{t} v^{n}+\partial_{x} f\left(v^{n}\right)+\partial_{x} P^{n-1}=0, & x \in \mathbb{R}, 0<t<\Delta t \\ P^{n-1}-\partial_{x x}^{2} P^{n-1}=3 f\left(u^{n-1}\right), & x \in \mathbb{R} \\ v^{n}(x, 0)=u^{n-1}(x), & x \in \mathbb{R}\end{cases}
$$

and

$$
u^{n}(\cdot):=v^{n}(\cdot, \Delta t) .
$$

We initialize by setting $u^{0}=u_{0}$. In the following we show that this sequence converges to an entropy solution of (3.1) as $\Delta t \rightarrow 0$, see Definition 1.1. To do this we establish "the usual estimates".

Lemma 3.1. Assume that (2.2) and (2.3) hold. Then

$$
\begin{aligned}
\left\|u^{n}\right\|_{L^{1}(\mathbb{R})} & \leq e^{3 L T}\left\|u_{0}\right\|_{L^{1}(\mathbb{R})}, \\
\left\|u^{n}\right\|_{L^{\infty}(\mathbb{R})} \leq\left|u^{n}\right|_{B V(\mathbb{R})} & \leq\left|u_{0}\right|_{B V(\mathbb{R})}+6 L T e^{3 L T}\left\|u_{0}\right\|_{L^{1}(\mathbb{R})} .
\end{aligned}
$$

for each $n \in\{0, \ldots, N\}$. Additionally, there exists a constant $C_{T, L}$, independent of the discretization parameters, such that

$$
\left\|u^{n}-u^{m}\right\|_{L^{1}(\mathbb{R})} \leq C_{T, L} \Delta t|n-m|, \quad n, m \in\{0, \ldots, N\} .
$$


Proof. Due to (2.8),

$$
\left\|v^{n}(\cdot, t)\right\|_{L^{1}(\mathbb{R})} \leq(1+3 L t)\left\|u^{n-1}\right\|_{L^{1}(\mathbb{R})}, \quad 0 \leq t \leq \Delta t,
$$

hence, from (3.3),

$$
\left\|u^{n}\right\|_{L^{1}(\mathbb{R})} \leq(1+3 L \Delta t)\left\|u^{n-1}\right\|_{L^{1}(\mathbb{R})},
$$

which implies (3.4).

We continue by proving (3.5). By (2.9),

$$
\left|v^{n}(\cdot, t)\right|_{B V(\mathbb{R})} \leq\left|u^{n-1}\right|_{B V(\mathbb{R})}+6 L t\left\|u^{n-1}\right\|_{L^{1}(\mathbb{R})}, \quad 0 \leq t \leq \Delta t,
$$

hence, from (3.3),

$$
\left|u^{n}\right|_{B V(\mathbb{R})} \leq\left|u^{n-1}\right|_{B V(\mathbb{R})}+6 L \Delta t\left\|u^{n-1}\right\|_{L^{1}(\mathbb{R})} .
$$

Therefore,

$$
\begin{aligned}
\left|u^{n}\right|_{B V(\mathbb{R})} & \leq\left|u_{0}\right|_{B V(\mathbb{R})}+6 L \Delta t \sum_{k=0}^{n-1}\left\|u^{k}\right\|_{L^{1}(\mathbb{R})} \\
& \leq\left|u_{0}\right|_{B V(\mathbb{R})}+6 L T e^{3 L T}\left\|u_{0}\right\|_{L^{1}(\mathbb{R})},
\end{aligned}
$$

which is (3.5).

Finally, (3.6) is a consequence of (3.5) and the fact that the "source" $\partial_{x} P^{n-1}$ in (3.2) is bounded in $L^{\infty}$, see (2.4).

For the convergence analysis, we introduce an approximation defined for all $t$ by

$$
u_{\Delta t}(\cdot, t):=u^{n}, \quad n \Delta t \leq t<(n+1) \Delta t, n \in\{0, \ldots, N-1\} .
$$

Theorem 3.1. Assume that (2.2) and (2.3) hold. Then as $\Delta t \rightarrow 0$

$$
u_{\Delta t} \rightarrow u \text { in } L^{p}(\mathbb{R} \times[0, T]) \forall p<\infty \text { and a.e. in } \mathbb{R} \times[0, T],
$$

where $u$ is the unique entropy solution to (3.1) in the sense of Definition 1.1.

Proof. By (3.4) and (3.5),

$$
\begin{aligned}
\left\|u_{\Delta t}(\cdot, t)\right\|_{L^{1}(\mathbb{R})} & \leq e^{3 L T}\left\|u_{0}\right\|_{L^{1}(\mathbb{R})}, \\
\left\|u_{\Delta t}(\cdot, t)\right\|_{L^{\infty}(\mathbb{R})} \leq\left\|u_{\Delta t}(\cdot, t)\right\|_{B V(\mathbb{R})} & \leq\left|u_{0}\right|_{B V(\mathbb{R})}+6 L T e^{3 L T}\left\|u_{0}\right\|_{L^{1}(\mathbb{R})},
\end{aligned}
$$

for any $0 \leq t \leq T$. Additionally, due to (3.6), there exists a constant $C_{T, L}$, independent of the discretization parameters, such that

$$
\left\|u_{\Delta t}(\cdot, t)-u_{\Delta t}(\cdot, s)\right\|_{L^{1}(\mathbb{R})} \leq C_{T, L}|t-s|, \quad t, s \in[0, T] .
$$

Using a standard argument (see, e.g., [9]), there exists a limit function $u$ and a sequence $\left\{\Delta t_{l}\right\}_{l}$, with $\Delta t_{l} \rightarrow 0$ as $l \rightarrow \infty$, such that

(3.11) $u_{\Delta t_{l}} \rightarrow u$ in $L^{p}(\mathbb{R} \times[0, T]) \forall p<\infty$ and a.e. in $\mathbb{R} \times[0, T]$,

$$
\|u(\cdot, t)\|_{L^{1}(\mathbb{R})},\|u(\cdot, t)\|_{L^{\infty}(\mathbb{R})},|u(\cdot, t)|_{B V(\mathbb{R})} \leq C_{T, L}, \quad \text { for each } t \in(0, T),
$$

where $C_{T, L}$ is a finite constant. In addition, $u \in C\left([0, T] ; L^{1}(\mathbb{R})\right)$.

To continue, we introduce an auxiliary function $v_{\Delta t}(x, t)$ defined by

$$
v_{\Delta t}(\cdot, t):=v^{n}(\cdot, t-n \Delta t),(n-1) \Delta t<t \leq n \Delta t, n \in\{1, \ldots, N\},
$$

where $v^{n}$ solves (3.2). Using (3.4), (3.5), and (3.10), there exists a constant $C_{T, L}$, independent of the discretization parameters, such that for all $t, s \in[0, T]$

$$
\left\|v_{\Delta t}(\cdot, t)\right\|_{L^{1}(\mathbb{R})} \leq C_{T, L},
$$




$$
\begin{aligned}
& \left\|v_{\Delta t}(\cdot, t)\right\|_{L^{\infty}(\mathbb{R})} \leq\left|v_{\Delta t}(\cdot, t)\right|_{B V(\mathbb{R})} \leq C_{L, T}, \\
& \left\|v_{\Delta t}(\cdot, t)-v_{\Delta t}(\cdot, t)\right\|_{L^{1}(\mathbb{R})} \leq C_{T, L}|t-s| .
\end{aligned}
$$

We claim that

$$
\left\|u_{\Delta t}(\cdot, t)-v_{\Delta t}(\cdot, t)\right\|_{L^{1}(\mathbb{R})} \rightarrow 0, \quad \text { as } \Delta t \rightarrow 0, \text { for each } 0 \leq t \leq T .
$$

To this end, let $t \in[(n-1) \Delta t, n \Delta t)$. Then, since $v^{n}(\cdot, 0)=u^{n-1}$ and thanks to (3.10) and (3.15),

$$
\begin{aligned}
\left\|u_{\Delta t}(\cdot, t)-v_{\Delta t}(\cdot, t)\right\|_{L^{1}(\mathbb{R})} & =\left\|u^{n-1}-v^{n}(\cdot, t-n \Delta t)\right\|_{L^{1}(\mathbb{R})} \\
& =\left\|v^{n}(\cdot, 0)-v^{n}(\cdot, t-n \Delta t)\right\|_{L^{1}(\mathbb{R})} \\
& \leq C_{T, L}(t-n \Delta t) \leq C_{T, L} \Delta t
\end{aligned}
$$

which proves (3.16). Thus $v_{\Delta t}$ it is the unique entropy solution to

$$
\begin{cases}\partial_{t} v_{\Delta t}+\partial_{x} f\left(v_{\Delta t}\right)+\partial_{x} P_{\Delta t}=0, & x \in \mathbb{R}, 0<t<T, \\ P_{\Delta t}-\partial_{x x}^{2} P_{\Delta t}=3 f\left(u_{\Delta t}\right), & x \in \mathbb{R}, 0<t<T \\ v_{\Delta t}(x, 0)=u_{0}(x), & x \in \mathbb{R} .\end{cases}
$$

Hence, for any convex entropy/entropy flux pair $(\eta, q)$ and for any nonnegative test function $\phi \in C_{c}^{\infty}(\mathbb{R} \times[0, T))$ we have, referring to (3.11) for the converging subsequence $\left\{\Delta t_{l}\right\}$,

$$
\begin{array}{r}
\int_{0}^{T} \int_{\mathbb{R}}\left(\eta\left(v_{\Delta t_{l}}\right) \partial_{t} \phi+q\left(v_{\Delta t_{l}}\right) \partial_{x} \phi-\eta^{\prime}\left(v_{\Delta t_{l}}\right) \partial_{x} P_{\Delta t_{l}} \phi\right) d x d t \\
+\int_{\mathbb{R}} \eta\left(u_{0}(x)\right) \phi(0, x) d x \geq 0 .
\end{array}
$$

By (3.11) and (3.16),

$$
v_{\Delta t_{l}} \rightarrow u \text { a.e. in } \mathbb{R} \times[0, T] \text { as } l \rightarrow \infty .
$$

Due to (3.11), (3.12), and the fact that

$$
P_{\Delta t_{l}}(x, t)=\frac{3}{2} \int_{\mathbb{R}} e^{-|x-y|} f\left(u_{\Delta t_{l}}(y, t)\right) d y,
$$

we conclude

$$
\partial_{x} P_{\Delta t_{l}} \rightarrow \partial_{x} P \text { a.e. in } \mathbb{R} \times[0, T] \text { as } l \rightarrow \infty,
$$

where $P-\partial_{x x}^{2} P=3 f(u)$.

Using (3.9), (3.14), (3.19), (3.20) and the dominated convergence theorem, sending $l \rightarrow \infty$ in (3.18) yields that the limit $u$ satisfies

$$
\int_{0}^{T} \int_{\mathbb{R}}\left(\eta(u) \partial_{t} \phi+q(u) \partial_{x} \phi-\eta^{\prime}(u) \partial_{x} P^{u} \phi\right) d x d t+\int_{\mathbb{R}} \eta\left(u_{0}(x)\right) \phi(0, x) d x \geq 0 .
$$

Then $u$ is the entropy solution to (3.1) in the sense of Definition 1.1. Finally, convergence of the whole sequence $\left\{u_{\Delta t}\right\}_{\Delta t>0}$ follows from the uniqueness of the entropy solution to (3.1) (see Theorem 1.1). 
3.2. Fully discrete schemes. In this subsection we introduce and analyze fully discrete schemes for (3.2). To this end, we introduce a time step $\Delta t>0$ and a local time step $\Delta \tau<\Delta t$ and let $N(\Delta t)=N \in \mathbb{N}, M(\Delta t, \Delta \tau)=M \in \mathbb{N}$ be the smallest integers satisfying

$$
M \Delta \tau=\Delta t, \quad N \Delta t=T
$$

Define

$$
U_{j}^{0}:=\frac{1}{\Delta x} \int_{\left(j-\frac{1}{2}\right) \Delta x}^{\left(j+\frac{1}{2}\right) \Delta x} u_{0}(x) d x, \quad j \in \mathbb{Z},
$$

and for $n \in\{1, \ldots, N\}$

$$
\begin{aligned}
& \frac{V_{j}^{n, m+1}-V_{j}^{n, m}}{\Delta \tau}+D_{-} F\left(V^{n, m} ; j\right)+D P_{j}^{n-1}=0, \\
& -D_{+} D_{-} P_{j}^{n-1}+P_{j}^{n-1}=3 f\left(U_{j}^{n-1}\right), \\
& V_{j}^{n, 0}=U_{j}^{n-1},
\end{aligned}
$$

where $j \in \mathbb{Z}, m \in\{0, \ldots, M-1\}$, and

$$
U_{j}^{n}:=V_{j}^{n, M}, \quad j \in \mathbb{Z},
$$

where we use the notations introduced in Section 2.2. As before, we assume that $F$ is a consistent numerical flux such that the scheme for the conservation law (without $P$ ) would be monotone. Recall that this implies that a CFL-condition must hold.

Lemma 3.2. Assume that (2.2), (2.3), and (2.12) hold. Then

$$
\begin{gathered}
\left\|\left\{U_{j}^{n}\right\}_{j}\right\|_{\ell^{1}} \leq e^{C_{1} L T}\left\|u_{0}\right\|_{L^{1}(\mathbb{R})}, \\
\left\|\left\{U_{j}^{n}\right\}_{j}\right\|_{\ell^{\infty}} \leq\left|\left\{U_{j}^{n}\right\}_{j}\right|_{b v} \leq\left|\left\{U_{j}^{0}\right\}_{j}\right|_{b v}+C_{1} L T e^{C_{1} L T}\left\|u_{0}\right\|_{L^{1}(\mathbb{R})} .
\end{gathered}
$$

for each $n \in\{0, \ldots, N\}$. Additionally, there exists a constant $C_{T, L}$, independent of the discretization parameters, such that

$$
\left\|\left\{U^{n}\right\} .-\left\{U^{m}\right\} .\right\|_{\ell^{1}} \leq C_{T, L} \Delta t|n-m|, \quad n, m \in\{0, \ldots, N\} .
$$

Proof. We begin by proving (3.25). By (2.21) we have

$$
\left\|\left\{V_{j}^{n, m}\right\}_{j}\right\|_{\ell^{1}} \leq\left(1+C_{1} L m \Delta \tau\right)\left\|\left\{U_{j}^{n-1}\right\}_{j}\right\|_{\ell^{1}}, \quad 0 \leq m \leq M-1 .
$$

Hence, from (3.21), (3.24),

$$
\left\|\left\{U_{j}^{n}\right\}_{j}\right\|_{\ell^{1}} \leq\left(1+C_{1} L M \Delta \tau\right)\left\|\left\{U_{j}^{n-1}\right\}_{j}\right\|_{\ell^{1}}=\left(1+C_{1} L \Delta t\right)\left\|\left\{U_{j}^{n-1}\right\}_{j}\right\|_{\ell^{1}} .
$$

Thus (3.25) holds. We continue by proving (3.26). Due to (2.22) we have

$$
\left|\left\{V_{j}^{n, m}\right\}_{j}\right|_{b v} \leq\left|\left\{U_{j}^{n-1}\right\}_{j}\right|_{b v}+C_{1} L m \Delta \tau\left\|\left\{U_{j}^{n-1}\right\}_{j}\right\|_{\ell^{1}},
$$

so, from (3.21) and (3.24), we obtain

$$
\begin{aligned}
\left|\left\{U_{j}^{n}\right\}_{j}\right|_{b v} & \leq\left|\left\{U_{j}^{n-1}\right\}_{j}\right|_{b v}+C_{1} L M \Delta \tau\left\|\left\{U_{j}^{n-1}\right\}_{j}\right\|_{\ell^{1}} \\
& =\left|\left\{U_{j}^{n-1}\right\}_{j}\right|_{b v}+C_{1} L \Delta t\left\|\left\{U_{j}^{n-1}\right\}_{j}\right\|_{\ell^{1}} .
\end{aligned}
$$

Now (3.26) follows as in the proof of Lemma 3.1. 
The proof of (3.27) is a consequence of the following calculation:

$$
\begin{aligned}
& \Delta x \sum_{i=0}^{m} \sum_{j=-\infty}^{\infty}\left|V_{j}^{n, i}-V_{j}^{n, i+1}\right| \\
& \leq \Delta x \lambda \sum_{i=0}^{m} \sum_{j=-\infty}^{\infty}\left|F\left(V_{j-p}^{n, i}, \ldots, V_{j+q}^{n, i}\right)-F\left(V_{j-p-1}^{n, i}, \ldots, V_{j+q-1}^{n, i}\right)\right| \\
& \quad+\Delta x \Delta \tau m \sum_{j=-\infty}^{\infty}\left|D P_{j}^{n}\right| \\
& \leq c_{1} \Delta \tau \sup _{i}\left|\left\{V_{j}^{n, i}\right\}_{j}\right|_{b v}+\Delta \tau m\left\|\left\{D P_{j}^{n-1}\right\}_{j}\right\|_{\ell^{1}} \\
& \leq c_{1} \Delta \tau\left(\left|\left\{U_{j}^{0}\right\}_{j}\right|_{B V}+C_{1} L T e^{C_{1} L T}\left\|u_{0}\right\|_{L^{1}(\mathbb{R})}\right)+m \Delta \tau C_{1} L e^{C_{1} L T}\left\|u_{0}\right\|_{L^{1}(\mathbb{R})},
\end{aligned}
$$

for any $n \in\{1, \ldots, N\}, m \in\{0, \ldots, m-1\}$.

Set $\Delta=(\Delta t, \Delta \tau)$, and define

$$
u_{\Delta}(x, t)=\sum_{j, n} I_{j}(x) I^{n}(t) U_{j}^{n},
$$

where $I^{n}$ now denotes the characteristic function of the interval $[n \Delta t,(n+1) \Delta t)$ and $I_{j}(x)$ is still the characteristic function of the interval $[(j-1 / 2) \Delta x,(j+1 / 2) \Delta x)$.

Theorem 3.2. Assume (2.2), (2.3), and (2.12) hold. Then as $\Delta t, \Delta \tau \rightarrow 0$, with $\frac{\Delta \tau}{\Delta t} \rightarrow 0$,

$$
u_{\Delta} \rightarrow u \text { in } L^{p}(\mathbb{R} \times[0, T]) \forall p<\infty \text { and a.e. in } \mathbb{R} \times[0, T],
$$

where $u$ is the unique entropy solution to (3.1) in the sense of Definition 1.1.

Proof. Due to $(3.25),(3.26)$,

$$
\begin{gathered}
\left\|u_{\Delta}(\cdot, t)\right\|_{L^{1}(\mathbb{R})} \leq e^{C_{1} L T}\left\|u_{0}\right\|_{L^{1}(\mathbb{R})}, \\
\left\|u_{\Delta}(\cdot, t)\right\|_{L^{\infty}(\mathbb{R})} \leq\left|u_{\Delta}(\cdot, t)\right|_{B V(\mathbb{R})} \leq\left|\left\{U_{j}^{0}\right\}_{j}\right|_{b v}+C_{1} L T e^{C_{1} L T}\left\|u_{0}\right\|_{L^{1}(\mathbb{R})},
\end{gathered}
$$

for any $0 \leq t<T$. Additionally, due to (2.23), there exists a constant $C_{T, L}$, independent of the discretization parameters, such that

$$
\left\|u_{\Delta \tau}(\cdot, t)-u_{\Delta \tau}(\cdot, t)\right\|_{L^{1}(\mathbb{R})} \leq C_{T, L}|t-s|, \quad t, s \in[0, T] .
$$

Using a standard argument, there exists a limit function $u$ and two sequences $\left\{\Delta t_{l}\right\}_{l},\left\{\Delta \tau_{l}\right\}_{l}, \Delta_{l}:=\left(\Delta t_{l}, \Delta \tau_{l}\right) \rightarrow 0$ such that

$$
\begin{aligned}
& u_{\Delta_{l}} \rightarrow u \text { in } L^{p}(\mathbb{R} \times[0, T]) \forall p<\infty \text { and a.e. in } \mathbb{R} \times[0, T], \\
& \|u(\cdot, t)\|_{L^{1}(\mathbb{R})},\|u(\cdot, t)\|_{L^{\infty}(\mathbb{R})},|u(\cdot, t)|_{B V(\mathbb{R})} \leq C_{T, L}, \quad \text { for each } t \in(0, T),
\end{aligned}
$$

where $C_{T, L}$ is a finite constant. In addition, $u \in C\left([0, T] ; L^{1}(\mathbb{R})\right)$.

Define

$$
v_{\Delta}(x, t)=\sum_{n, m \geq 0, j \in \mathbb{Z}} I_{j}^{n}(x, t) J^{m}(t-n \Delta t) V_{j}^{n, m}
$$

with $J^{m}$ denoting the characteristic interval of $[m \Delta \tau,(m+1) \Delta \tau)$. Here we recall that $V_{j}^{n, m}$ solves (3.23). Using (3.25), (3.26), (3.27), (3.28), (3.29) we see that

$$
\left\|v_{\Delta}(\cdot, t)\right\|_{L^{1}(\mathbb{R})} \leq e^{C_{1} L T}\left\|u_{0}\right\|_{L^{1}(\mathbb{R})}
$$




$$
\left|v_{\Delta}(\cdot, t)\right|_{B V(\mathbb{R})} \leq\left|\left\{U_{j}^{0}\right\}_{j}\right|_{b v}+C_{1} L T e^{C_{1} L T}\left\|u_{0}\right\|_{L^{1}(\mathbb{R})},
$$

for any $0 \leq t<T$. We claim that

$$
\left\|u_{\Delta}(\cdot, t)-v_{\Delta}(\cdot, t)\right\|_{L^{1}(\mathbb{R})} \rightarrow 0, \quad \text { as } \Delta \rightarrow 0, \text { for each } 0 \leq t \leq T .
$$

Let $0 \leq t \leq T$ and $n \in\{0, \ldots, N-1\}, m \in\{0, \ldots, M-1\}$, such that

$$
n \Delta t+m \Delta \tau \leq t<n \Delta t+(m+1) \Delta \tau \text {. }
$$

For the sake of simplicity we consider only the case $m \neq 0, n \Delta t+m \Delta \tau<t<$ $n \Delta t+(m+1) \Delta \tau$. Now we find that (see the proof of (3.2))

$$
\begin{aligned}
\left\|u_{\Delta}(\cdot, t)-v_{\Delta}(\cdot, t)\right\|_{L^{1}(\mathbb{R})} & =\Delta x \sum_{j=-\infty}^{\infty}\left|V_{j}^{n+1,0}-V_{j}^{n+1, m+1}\right| \\
& \leq \Delta x \sum_{i=0}^{m} \sum_{j=-\infty}^{\infty}\left|V_{j}^{n+1, i}-V_{j}^{n+1, i+1}\right| \leq C_{T, L} \tau
\end{aligned}
$$

where $C_{T, L}>0$ independent of the discretization parameters, and (3.35) thus holds.

We continue by showing that $u$ is an entropy solution to (3.1). Let $k$ be any real number and define $Q_{k}, q_{k}$ as in the proof of Theorem 2.1, see (2.33) and (2.38).

Arguing as for (2.34) we get

$$
\frac{\left|V_{j}^{n, m+1}-k\right|-\left|V_{j}^{n, m}-k\right|}{\Delta \tau}+D_{-} Q_{k}\left(V^{n, m} ; j\right)+D P_{j}^{n-1} \operatorname{sign}\left(V_{j}^{n, m+1}-k\right) \leq 0 .
$$

For a fixed test function $\phi \in C_{c}^{\infty}(\mathbb{R} \times[0, T)), \phi \geq 0$, we compute

$$
\begin{aligned}
& \sum_{m=0}^{M-1} \sum_{n=0}^{N-1} \sum_{j=-\infty}^{\infty} \phi\left(j \Delta x, t_{n, m}\right) \frac{\left|V_{j}^{n, m+1}-k\right|-\left|V_{j}^{n, m}-k\right|}{\Delta \tau} \\
& \quad+\sum_{m=0}^{M-1} \sum_{n=0}^{N-1} \sum_{j=-\infty}^{\infty} \phi\left(j \Delta x, t_{n, m}\right) D_{-} Q_{k}\left(V^{n, m} ; j\right) \\
& \quad+\sum_{m=0}^{M-1} \sum_{n=0}^{N-1} \sum_{j=-\infty}^{\infty} \phi\left(j \Delta x, t_{n, m}\right) D P_{j}^{n-1} \operatorname{sign}\left(V_{j}^{n, m+1}-k\right) \leq 0,
\end{aligned}
$$

where $t_{n, m}:=n \Delta t+m \Delta \tau$. Summing by parts and using (3.21) produce

$$
\begin{aligned}
\sum_{m=0}^{M-1} & \sum_{n=0}^{N-1} \sum_{j=-\infty}^{\infty} \phi\left(j \Delta x, t_{n, m}\right) \frac{\left|V_{j}^{n, m+1}-k\right|-\left|V_{j}^{n, m}-k\right|}{\Delta \tau} \\
& -\sum_{m=1}^{M-1} \sum_{n=0}^{N-1} \sum_{j=-\infty}^{\infty} \frac{\phi\left(j \Delta x, t_{n, m}\right)-\phi\left(j \Delta x, t_{n, m-1}\right)}{\Delta \tau}\left|V_{j}^{n, m}-k\right| \\
& +\sum_{n=0}^{N-1} \sum_{j=-\infty}^{\infty} \phi\left(j \Delta x, t_{n, M-1}\right) \frac{\left|V_{j}^{n, M}-k\right|}{\Delta \tau} \\
& -\sum_{n=0}^{N-1} \sum_{j=-\infty}^{\infty} \phi\left(j \Delta x, t_{n, 0}\right) \frac{\left|V_{j}^{n, 0}-k\right|}{\Delta \tau} \\
=- & \sum_{m=1}^{M-1} \sum_{n=0}^{N-1} \sum_{j=-\infty}^{\infty} \frac{\phi\left(j \Delta x, t_{n, m}\right)-\phi\left(j \Delta x, t_{n, m-1}\right)}{\Delta \tau}\left|V_{j}^{n, m}-k\right|
\end{aligned}
$$




$$
\begin{aligned}
& +\sum_{n=0}^{N-1} \sum_{j=-\infty}^{\infty} \phi\left(j \Delta x, t_{n, M-1}\right) \frac{\left|V_{j}^{n+1,0}-k\right|}{\Delta \tau} \\
& -\sum_{n=0}^{N-1} \sum_{j=-\infty}^{\infty} \phi\left(j \Delta x, t_{n, 0}\right) \frac{\left|V_{j}^{n, 0}-k\right|}{\Delta \tau} \\
& =-\sum_{m=1}^{M-1} \sum_{n=0}^{N-1} \sum_{j=-\infty}^{\infty} \frac{\phi\left(j \Delta x, t_{n, m}\right)-\phi\left(j \Delta x, t_{n, m-1}\right)}{\Delta \tau}\left|V_{j}^{n, m}-k\right| \\
& +\sum_{n=0}^{N-1} \sum_{j=-\infty}^{\infty} \phi\left(j \Delta x, t_{n+1,-1}\right) \frac{\left|V_{j}^{n+1,0}-k\right|}{\Delta \tau} \\
& -\sum_{n=0}^{N-1} \sum_{j=-\infty}^{\infty} \phi\left(j \Delta x, t_{n, 0}\right) \frac{\left|V_{j}^{n, 0}-k\right|}{\Delta \tau} \\
& =-\sum_{m=1}^{M-1} \sum_{n=0}^{N-1} \sum_{j=-\infty}^{\infty} \frac{\phi\left(j \Delta x, t_{n, m}\right)-\phi\left(j \Delta x, t_{n, m-1}\right)}{\Delta \tau}\left|V_{j}^{n, m}-k\right| \\
& +\sum_{n=1}^{N-1} \sum_{j=-\infty}^{\infty} \frac{\phi(j \Delta x, n \Delta t-\Delta \tau)-\phi(j \Delta x, n \Delta t)}{\Delta \tau}\left|V_{j}^{n, 0}-k\right| \\
& +\sum_{j=-\infty}^{\infty} \frac{\phi(j \Delta x, N \Delta t-\Delta \tau)}{\Delta \tau}\left|V_{j}^{N, 0}-k\right| \\
& -\sum_{j=-\infty}^{\infty} \phi(j \Delta x, 0) \frac{\left|U_{j}^{0}-k\right|}{\Delta \tau} \\
& \sum_{m=0}^{M-1} \sum_{n=0}^{N-1} \sum_{j=-\infty}^{\infty} \phi\left(j \Delta x, t_{n, m}\right) D_{-} Q_{k}\left(V^{n, m} ; j\right) \\
& =-\sum_{m=0}^{M-1} \sum_{n=0}^{N-1} \sum_{j=-\infty}^{\infty} Q_{k}\left(V^{n, m} ; j\right) D_{+} \phi\left(j \Delta x, t_{n, m}\right),
\end{aligned}
$$

hence, due to the identity $N \Delta t-\Delta \tau=T-\Delta \tau$ (see (3.21)), (3.36) becomes

$$
\begin{aligned}
& -\Delta x \Delta \tau \sum_{m=1}^{M-1} \sum_{n=0}^{N-1} \sum_{j=-\infty}^{\infty} \frac{\phi\left(j \Delta x, t_{n, m}\right)-\phi\left(j \Delta x, t_{n, m-1}\right)}{\Delta \tau}\left|V_{j}^{n, m}-k\right| \\
& +\Delta x \Delta \tau \sum_{n=1}^{N-1} \sum_{j=-\infty}^{\infty} \frac{\phi(j \Delta x, n \Delta t-\Delta \tau)-\phi(j \Delta x, n \Delta t)}{\Delta \tau}\left|V_{j}^{n, 0}-k\right| \\
& +\Delta x \sum_{j=-\infty}^{\infty} \phi(j \Delta x, T-\Delta \tau)\left|V_{j}^{N, 0}-k\right| \\
& -\Delta x \sum_{j=-\infty}^{\infty} \phi(j \Delta x, 0)\left|U_{j}^{0}-k\right|
\end{aligned}
$$




$$
\begin{aligned}
& -\Delta x \Delta \tau \sum_{m=0}^{M-1} \sum_{n=0}^{N-1} \sum_{j=-\infty}^{\infty} Q_{k}\left(V^{n, m} ; j\right) D_{+} \phi\left(j \Delta x, t_{n, m}\right) \\
& +\Delta x \Delta \tau \sum_{m=0}^{M-1} \sum_{n=0}^{N-1} \sum_{j=-\infty}^{\infty} \phi\left(j \Delta x, t_{n, m}\right) D P_{j}^{n} \operatorname{sign}\left(V_{j}^{n, m+1}-k\right) \leq 0
\end{aligned}
$$

Since $\operatorname{supp}(\phi)$ is a compact subset of $\mathbb{R} \times[0, T)$, we have $(j \Delta x, T-\Delta \tau) \notin \operatorname{supp}(\phi)$ for $\Delta \tau$ small enough, and since $V^{N, 0}$ is bounded we find that

$$
\Delta x \sum_{j=-\infty}^{\infty} \phi(j \Delta x, T-\Delta \tau)\left|V_{j}^{N, 0}-k\right| \rightarrow 0, \quad \text { as } \Delta t, \Delta \tau \rightarrow 0 .
$$

Furthermore

$$
\begin{aligned}
\Delta x \Delta \tau \sum_{n=1}^{N-1} \sum_{j=-\infty}^{\infty} & \frac{\phi(j \Delta x, n \Delta t-\Delta \tau)-\phi(j \Delta x, n \Delta t)}{\Delta \tau}\left|V_{j}^{n, 0}-k\right| \\
& \leq \frac{\Delta \tau}{\Delta t} T\left\|\left\{|| V_{j}^{n, 0}-k \mid\right\}_{j, n}\right\|_{\ell^{\infty}} \sup _{0 \leq t \leq T}\left\|\partial_{t} \phi(\cdot, t)\right\|_{L^{1}(\mathbb{R})} \rightarrow 0 .
\end{aligned}
$$

Next, thanks to the $b v$ estimate (3.29) and the consistency of the numerical flux,

$$
\Delta \tau \Delta x \sum_{m=0}^{M-1} \sum_{n=0}^{N-1} \sum_{j=-\infty}^{\infty}\left|Q_{k}\left(V^{n, m} ; j\right)-q_{k}\left(V_{j}^{n, m}\right)\right| \rightarrow 0, \quad \text { as } \Delta t, \Delta \tau \rightarrow 0 .
$$

As before, we have that

$$
D P_{\Delta t_{l}, \Delta \tau_{l}} \rightarrow \partial_{x} P \text { a.e. in } \mathbb{R} \times[0, T] \text { as } l \rightarrow \infty,
$$

where $P-\partial_{x x}^{2} P=3 f(u)$, and

$$
P_{\Delta t_{l}, \Delta \tau_{l}}(x, t)=\int^{x} \sum_{j} D P_{j}^{n} I_{j}^{n}(y, t) d y .
$$

Using (3.31)-(3.35), (3.38)-(3.41) and the dominated convergence theorem when sending $l \rightarrow \infty$ in (3.37), we find that the limit $u$ satisfies the entropy inequality (1.5) for all $k \in \mathbb{R} \backslash \Theta$, where $\Theta$ is an at most countable set $\Theta$ (see the proof of Theorem 2.1). To conclude from this that (2.39) holds for all $k \in \mathbb{R}$, i.e., that $u$ is an entropy solution to (3.1) in the sense of Definition 1.1, we repeat the argument in [11]. Again, convergence of the whole sequence $\left\{u_{\Delta t}\right\}_{\Delta t>0}$ follows from the uniqueness of entropy solutions.

\section{NumericAL EXAMPLES}

In this section we test our scheme on two examples. In order to compare our results with an "exact" solution we use the shock-peakons described by Lundmark in [14]. These are exact entropy solutions to the Degasperis-Procesi equation (1.1) and are given by the formulas

$$
u(x, t)=\sum_{k=1}^{N}\left\{m_{k}(t)-s_{k}(t) \operatorname{sign}\left(x-x_{k}(t)\right)\right\} e^{-\left|x-x_{k}(t)\right|},
$$


where the coefficients $x_{k}, m_{k}$, and $s_{k}$ satisfy the following system of ordinary differential equations:

$$
\begin{aligned}
\frac{d}{d t} m_{k}(t)=2[ & s_{k}(t) u\left(x_{k}, t\right) \\
& \left.\quad-m_{k}(t) \sum_{j=1}^{N}\left(s_{j}(t)-\operatorname{sign}\left(x_{k}(t)-x_{j}(t)\right) m_{j}(t)\right) e^{-\left|x_{k}(t)-x_{j}(t)\right|}\right], \\
\frac{d}{d t} s_{k}(t)=- & \left.s_{k}(t)\right) \sum_{j=1}^{N}\left(s_{j}(t)-\operatorname{sign}\left(x_{k}(t)-x_{j}(t)\right) m_{j}(t)\right) e^{-\left|x_{k}(t)-x_{j}(t)\right|} .
\end{aligned}
$$

Unless $N=1$ it is not possible to find explicit solutions to this system of equations, and we used Matlab's routine ode23s (after some trials) to integrate the system numerically. The result of this was then used as an exact solution with which we compared the approximations calculated with our numerical scheme.

If $N=1$ we have three different types of traveling waves, the peakon

$$
u(x, t)=e^{|x-t|}
$$

the antipeakon

and the shockpeakon

$$
u(x, t)=-e^{|x-t|},
$$

$$
u(x, t)=-\frac{\operatorname{sign}(x) e^{-|x|}}{1+t} .
$$

We have tested the scheme using the Engquist-Osher numerical flux function (2.13). For our test we used $N=3$ and the initial values

$$
\begin{array}{lll}
x_{1}(0)=-5, & x_{2}(0)=0, & x_{3}(0)=5, \\
m_{1}(0)=1, & m_{2}(0)=0, & m_{3}(0)=-1, \\
s_{1}(0)=0, & s_{2}(0)=1, & s_{3}(0)=0,
\end{array}
$$

which gives the initial function

$$
u_{0}(x)=e^{-|x+5|}+\operatorname{sign}(x) e^{-|x|}-e^{-|x-5|} .
$$

The exact solution will be a peakon, shockpeakon, antipeakon collision at $t=5$ and $x=0$. For $t>5$ the solution continues as a decaying shockpeakon.

We used $2^{M}$ uniformly spaced grid points in the interval $x \in[-10,10]$. The relationship between $\Delta \tau$ and $\Delta t$ were such that $(M / 4) \Delta \tau=\Delta t$, and $\Delta \tau$ was determined according to the CFL-condition

$$
\Delta \tau=0.5 \Delta x \max _{j}\left\{\left|U_{j}^{n}\right|\right\} .
$$

In Figure 1 we show the approximate solution obtained using $2^{8}$ gridpoints in $[-10,10]$ for $t \in[0,8]$. Since we have an (almost) exact solution to compare with, we tabulated the relative $\ell^{1}$ error, defined by

$$
\operatorname{err}=\max _{n} \frac{\sum_{j}\left|U_{j}^{n}-u_{j}^{n}\right|}{\sum_{j}\left|u_{j}^{n}\right|}
$$

where $u_{j}^{n}$ denotes the exact solution at $\left(x_{j}, t^{n}\right)$. In Table 1 we report these errors. 


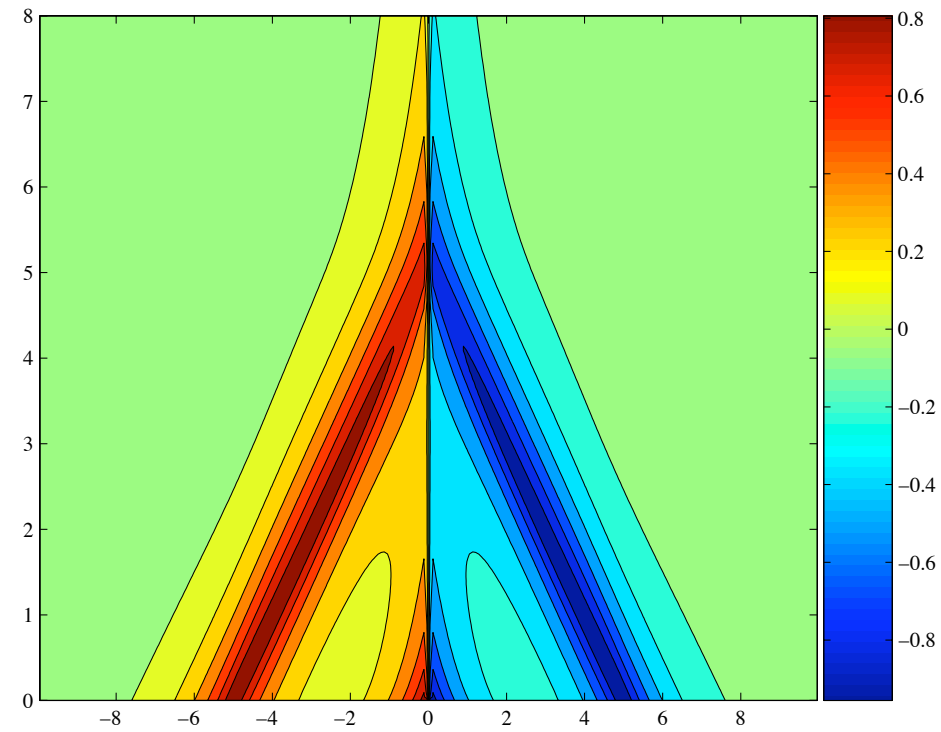

FiguRE 1. The approximation to with initial data (4.5) and 256 gridpoints in $[-10,10]$.

$$
\begin{array}{ccccccccc}
M & 4 & 5 & 6 & 7 & 8 & 9 & 10 & 11 \\
\text { err } & 3.35 & 1.10 & 0.51 & 0.39 & 0.28 & 0.17 & 0.11 & 0.07
\end{array}
$$

TABLE 1. Relative $\ell^{1}$ errors. Here $\Delta x=20 \times 2^{-M}$ and "err" is defined in (4.6). The initial data are given by (4.5).

This computations confirm the convergence established in the previous section, and give a numerical convergence rate of about $1 / 3$. We remark that the observed numerical convergence is improved if $\Delta \tau=\Delta t$, but we have not been able to prove that the resulting scheme converges.

For the solutions of the Degasperis-Procesi equation given by (4.1) shocks cannot form from continuous initial data. If $s_{k}=0$ for all $k$, then the third equation gives $s_{k}^{\prime}(t)=0$, and thus $s_{k}$ remains zero unless $m_{i}$ blows up. We observed such singular behavior numerically for peakon-antipeakon collisions, but it was not possible to integrate the system past the collision time. Despite this we observed that shock formation was generic if $u_{0}^{\prime}(x)<0$ and $u_{0}(x)<0$ for some $x$. To illustrate this we show a computed example where shocks form as they would in the conservation law. In this case the initial function is given by

$$
u_{0}(x)=e^{0.5 x^{2}} \sin (\pi x),
$$

for $x \in[-2,2]$, where we assume that $u_{0}$ is extended periodically outside this interval. In Figure 2 we show the computed solution using $2^{8}$ gridpoints in the 
interval $[-2,2]$ for $t \in[0,1.3]$. We see that the $N$-waves familiar from Burgers' equation do indeed form, even though the initial data is continuous.

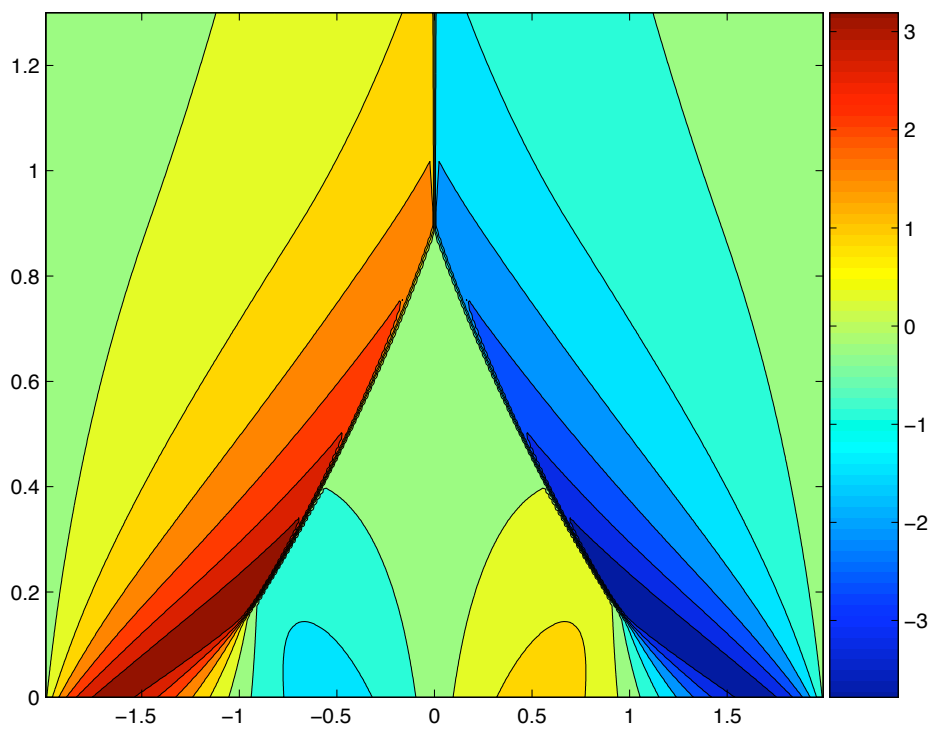

FIGURE 2. An approximate solution with initial data (4.7).

\section{REFERENCES}

[1] F. Bratvedt, K. Bratvedt, C. F. Buchholz, T. Gimse, H. Holden, L. Holden, and N. H. Risebro. FRONTLINE and FRONTSIM: two full scale, two-phase, black oil reservoir simulators based on front tracking. Surveys Math. Indust., 3(3):185-215, 1993.

[2] R. Camassa and D. D. Holm. An integrable shallow water equation with peaked solitons. Phys. Rev. Lett., 71(11):1661-1664, 1993.

[3] Z. Chen. Degenerate two-phase incompressible flow. I. Existence, uniqueness and regularity of a weak solution. J. Differential Equations, 171(2):203-232, 2001.

[4] G. M. Coclite and K. H. Karlsen. On the well-posedness of the Degasperis-Procesi equation. J. Funct. Anal., 233(1):60-91, 2006.

[5] A. Constantin and L. Molinet. Global weak solutions for a shallow water equation. Comm. Math. Phys., 211(1):45-61, 2000.

[6] A. Degasperis, D. D. Holm, and A. N. W. Hone. Integrable and non-integrable equations with peakons. In Nonlinear physics: theory and experiment, II (Gallipoli, 2002), pages 3743. World Sci. Publishing, River Edge, NJ, 2003.

[7] A. Degasperis, D. D. Holm, and A. N. I. Khon. A new integrable equation with peakon solutions. Teoret. Mat. Fiz., 133(2):170-183, 2002.

[8] A. Degasperis and M. Procesi. Asymptotic integrability. In Symmetry and perturbation theory (Rome, 1998), pages 23-37. World Sci. Publishing, River Edge, NJ, 1999.

[9] H. Holden and N. H. Risebro. Front tracking for hyperbolic conservation laws, volume 152 of Applied Mathematical Sciences. Springer-Verlag, New York, 2002.

[10] D. D. Holm and M. F. Staley. Wave structure and nonlinear balances in a family of evolutionary PDEs. SIAM J. Appl. Dyn. Syst., 2(3):323-380 (electronic), 2003.

[11] K. H. Karlsen, N. H. Risebro, and J. D. Towers. Front tracking for scalar balance equations. J. Hyperbolic Differ. Equ., 1(1):115-148, 2004. 
[12] C. Lattanzio and P. Marcati. Global well-posedness and relaxation limits of a model for radiating gas. J. Differential Equations, 190(2):439-465, 2003.

[13] H. Liu and E. Tadmor. Critical thresholds in a convolution model for nonlinear conservation laws. SIAM J. Math. Anal., 33(4):930-945 (electronic), 2001.

[14] H. Lundmark. Formation and dynamics of shock waves in the Degasperis-Procesi equation. Preprint, 2006.

[15] H. Lundmark and J. Szmigielski. Multi-peakon solutions of the Degasperis-Procesi equation. Inverse Problems, 19(6):1241-1245, 2003.

[16] H. Lundmark and J. Szmigielski. Degasperis-Procesi peakons and the discrete cubic string. IMRP Int. Math. Res. Pap., (2):53-116, 2005.

[17] O. G. Mustafa. A note on the Degasperis-Procesi equation. J. Nonlinear Math. Phys., 12(1):10-14, 2005.

[18] D. Serre. $L^{1}$-stability of constants in a model for radiating gases. Commun. Math. Sci., 1(1):197-205, 2003.

[19] Z. Xin and P. Zhang. On the weak solutions to a shallow water equation. Comm. Pure Appl. Math., 53(11):1411-1433, 2000.

[20] Z. Yin. Global existence for a new periodic integrable equation. J. Math. Anal. Appl., 283(1):129-139, 2003.

[21] Z. Yin. On the Cauchy problem for an integrable equation with peakon solutions. Illinois J. Math., 47(3):649-666, 2003.

[22] Z. Yin. Global solutions to a new integrable equation with peakons. Indiana Univ. Math. J., 53(4):1189-1209, 2004.

[23] Z. Yin. Global weak solutions for a new periodic integrable equation with peakon solutions. J. Funct. Anal., 212(1):182-194, 2004.

(Giuseppe Maria Coclite)

DePARTMENT OF MATHEMATICS, UNiversity OF BARI

Via E. Orabona 4, 70125 Bari, Italy

E-mail address: coclitegm@dm.uniba.it

(Kenneth Hvistendahl Karlsen)

Centre of Mathematics for Applications (CMA), University of Oslo

P.O. Box 1053, Blindern N-0316 Oslo, Norway

E-mail address: kennethk@math.uio.no

$U R L:$ http://www.math.uio.no/ kennethk/

(Nils Henrik Risebro)

Centre of Mathematics for Applications (CMA), University of Oslo

P.O. Box 1053, Blindern, N-0316 Oslo, Norway

E-mail address: nilshr@math.uio.no

URL: http://www.math.uio.no/〜nilshr/ 